\title{
Quantitative Study Designs: Experimental
}

Correlation is not causation.

—Karl Pearson, mathematician

\section{LEARNING OBJECTIVES}

After reading this chapter, the reader will be able to

- Explain the concept of causality.

- Define internal validity.

- Identify the major threats to internal validity.

- Describe four types of experimental study designs.

- Develop study designs to achieve research objectives.

\section{INTRODUCTION}

There are many ways to study a public health issue. Consider the topic of electric cigarettes or vaping. You could explore the facilitators of vaping among adolescents, describe the correlates of use, explain the determinants of use, or evaluate the effectiveness of a program or policy to reduce this behavior. As stated in Chapter 1, The Importance of Research in Public Health, the main purpose of research (exploration, description, explanation, and evaluation) informs the ways a study is planned and implemented. The purpose drives study design. For example, explorative and descriptive studies typically do not rely on proving cause and effect. However, 
if the research purpose is to explain or fully evaluate the effectiveness of a program or policy, certain study design requirements have to be met. Suppose your research question is "Does the tailored stress-management intervention improve sleep health in adults?" You use probability sampling to identify a sample of 100 people already enrolled in a large stress-management program. You randomly assign 50 of them to get a tailored sleep health intervention as part of stress management. The other 50 people get the usual intervention. If the intervention group reports better sleep duration and quality compared to the usual care group, we would be able to infer the tailored sleep health intervention was the reason for the difference. In this simplified description of the sleep health study, we need to determine that it was, in fact, the intervention and nothing else that contributed to the difference between groups in the outcome measure. The type of study design influences the level of certainty in making this inference.

In order to conclude the independent variable (intervention) caused the change to our dependent variable (sleep duration and quality), the three criteria for causal inference must be met. Although with some variation, there is a fair amount of consensus in the scientific community for three criteria used for causal inference in general research. The first criterion for inferring causality is association. The independent and dependent variables must covary with one another or be associated in some way. A change in one will result in a change in the other. Without an association, there can be no causal relationship. The second criterion for inferring causality is order in time. The cause must precede the effect. This may seem obvious, but it needs to be validated. Suppose you find that most people who washed their hands throughout the day watched a popular movie about a deadly flu outbreak and most people who reported not washing their hands throughout the day did not watch the movie. You think there is an association between handwashing and the movie, only to find out the handwashing data was collected before the movie was released. Because the dependent variable (handwashing) occurred before the independent variable (the movie), we cannot infer that watching the movie caused the difference in handwashing behavior. The third criterion for inferring causality is nonspuriousness. This means the covariation between the two variables cannot be explained as the result of influence of some other variable. The association between two variables might be caused by something else. We need to design our studies in a way to ensure the only influence on the dependent variable is the independent variable and the variation is not due to a third (i.e., extraneous) variable.

To recap, the three criteria for inferring causality are

1. the variables must be associated or covary,

2. the cause must precede the effect in time, and

3. the relationship cannot be explained by a third variable of influence.

\section{INTERNAL VALIDITY}

Chapter 4, Operationalization and Measurement, describes internal validity as the extent to which a measure accurately depicts what it is intended to measure. Related to causal inference, internal validity means the study results accurately represent causation. This concept refers to the confidence that the three criteria for inferring causality are met. In an internally valid study, you are sure the two variables are associated, are in the correct time order, and no third variable contributed to the association. Some aspects of a study can interfere with internal validity. There are several threats to internal validity common in public health research. Careful study design, implementation, and data analysis can reduce the risk and impact of these threats. 


\section{Selection Bias}

Chapter 5, Sampling, defines sampling bias, or creating a sample that is not representative due to a variety of factors. Selection bias is different. Although sample bias means the sample may be different from the target population, this type of bias occurs when the groups in your study are not comparable. In the previous sleep health intervention example, what if you asked for volunteers to participate in the intervention and put all those who refused into the control group? We cannot be certain the improvement in sleep duration and quality in the intervention group was not due to characteristics of people in each group. The groups were not comparable. Random assignment into the intervention and control groups is one way to reduce selection bias. In many public health research studies, however, random assignment may not be feasible or ethical. Imagine studying the impact of some environmental change. One community in the study area is exposed to the change, while the other is not. You cannot randomly place residents into communities to fit your study design. In this example the criteria for inferring causality are not met, and because of nonrandom assignment, a third variable (e.g., participant characteristic) might influence the outcome. Causality is an important outcome of research, but it is not the only reason to conduct studies. There still is value in findings from studies that do not meet the criteria for causality. Quasi-experimental studies, as discussed in Chapter 7, Quantitative Study Designs: Nonexperimental, make valid contributions to public health evidence.

\section{History}

This bias does not refer to things you might have learned in high school history class. Events occurring during the course of your research study can impact the outcome. Suppose you implement a distracted-driving awareness intervention in a community. During the intervention, one of the town's elected officials is involved in a crash caused by texting while driving. The story is published in all of the local news outlets. The change in awareness of the risks of distracted driving may not have been solely due to your intervention. People may have been made more aware of the issue from the news story. Although you do not have control over such extraneous events, they need to be reported as a limitation when you write up the study results.

In 1998, the husband of well-known news celebrity Katie Couric died of colon cancer. He was 42. Ms. Couric was devastated by his death and became a fierce advocate for awareness of this disease. In March 2000, she underwent a live on-air colonoscopy on the Today Show as part of a weeklong focus on colon cancer awareness and the importance of colon cancer screening. The result was a temporary increase in colonoscopy use. Dubbed the "Katie Couric effect," research findings on this topic suggest a celebrity spokesperson can have a substantial impact on public participation in preventive-care programs. This also poses an example of how extraneous events can impact research outcomes. The death of her husband and Ms. Couric's advocacy could have been a threat to internal validity of any other colon cancer intervention going on at the same time. ${ }^{11}$ 


\section{Testing}

Testing can contribute to improved performance in and of itself. Think about how much time and effort some high school students put into practicing for the American College Testing (ACT) or Scholastic Assessment Test (SAT) exams. They take the tests over and over to increase their scores. Their knowledge of the subjects may not increase, but their testtaking skills improve. Sometimes in research, the process of testing or assessment creates improvement, not necessarily due to improvements in the construct it is measuring. This may result in failure to find any significant intervention effects because both intervention and control groups achieved better outcomes due to testing effects. Assessments can act as an intervention. Suppose you design a weight-loss study with randomly selected intervention and control groups. Weighing the control group participants at baseline might incite behaviors conducive to weight loss, even if they are not exposed to the intervention. Certain study designs can be implemented to rule out testing effects.

\section{Maturation}

Research studies are implemented over time, and the effect of time may produce short- or long-term changes in the participants of your study. These changes can impact your results. Short-term maturation effects may be due to changes in mood of the participant because of tiredness or boredom. Although a researcher may not be able to control how much sleep a participant gets before the intervention occurs, they can try to reduce participant burden and consider potential short-term maturation effects in study design. Long-term maturation changes include aging, advancing in education, or increasing/decreasing income. These changes can occur within time frames of a few months to years. Think about how much growth and development happens during puberty. A long-term study on food intake and weight on young adolescents would definitely have to consider the potential effects of maturation. Employment, childbirth, and retirement can contribute to maturation effects in adults. Older adults may experience physical or cognitive changes during the time period of a study. Reviewing the literature and fully exploring your research topic will help determine the potential extent of maturation as a threat to internal validity.

\section{Attrition}

If participants drop out before study completion, this may affect study outcome. In longer term health studies, mortality may reduce the study sample at posttest. Attrition can be a threat to internal validity when those who drop out are significantly different from participants remaining in the study. For example, in a 12-week intervention to prevent cardiovascular disease, $40 \%$ of participants drop out after week 6 . Upon inquiry, you find out most of those who dropped out were laid off from their jobs at a local employer when a factory shut down. The characteristic (employment) between those who remained in class versus those who dropped out can be a significant limitation to study findings. At the end of your study, you can statistically compare characteristics of those who dropped out with those who remained in the study in order to identify attrition bias. High attrition may also cause 
analysis difficulties, especially if you need a minimum group number to determine statistical significance. As a preventive measure, make sure the sample is large enough to accommodate potential attrition. Information from past studies may provide helpful information about attrition in specific population groups.

There are several ways to reduce the potential for attrition bias. The use of incentives, or providing some type of compensation, is one strategy recommended to maintain participation in research studies. Graduated incentives may be effective in keeping people involved in longer term studies. With this method, participants are offered a choice of smaller incentives (typically monetary) at each intervention segment, or a larger incentive to be received at the end of the study. For example, in a 6-month evaluation of the impact of a campaign to reduce sugar-sweetened beverage intake among high school students, students might be offered $\$ 10$ after completing the baseline survey, $\$ 10$ at the 3-month survey, and $10 \$$ at the 6-month follow-up. The other incentive option might be to provide $\$ 50$ to each participant who completes the third survey at 6 months (versus a total of $\$ 30$ if they take the incentive in increments). Another strategy to reduce attrition is through follow-up with participants. You will need accurate contact information as well as preferred method of contact for effective follow-up, as well as dedicated research staff time for comprehensive follow-up.

\section{Statistical Regression}

Statistical regression as a threat to internal validity has to do with "room for improvement." When scores from assessment are particularly high or low, there is a tendency for these scores to move toward the mean or average score. Let's use physical activity as an example. People who are mostly sedentary (i.e., take less than 1,000 steps per day) will likely show improvement in a walking intervention because they have such low activity to begin with. If more sedentary people are in the intervention group, they will show greater improvement. Researchers can reduce the risk of statistical regression as a threat to internal validity by using random group assignment and reliable measures.

\section{Instrumentation Changes}

Internal validity may be compromised if the instrument or measuring device changes over time. These changes can be due to the researcher or the actual measuring device. For example, at the beginning of an intervention your study team takes photos to analyze outdoor smoking spaces. At the end of the intervention, your team conducts observations of the same spaces. There may be differences in the factors captured by these two methods. Consistency in the measuring instrument is important. Both photos and observations can be valid ways to assess the spaces, but may not be comparable. In addition, changes may happen to the measurement device over the course of the study. Suppose you use a tape measure to calculate the amount of space allotted to tobacco products in grocery stores. After the 25th store, the tape measure becomes stretched out. The measurements taken at the stores at the beginning might be slightly different from those measured later in the study. Study design should include consistent and precise measures. 


\section{THE HAWTHORNE EFFECT}

Just knowing research is being conducted may result in a change in study participants' behavior. This phenomenon is termed the Hawthorne effect after productivity experiments conducted between 1924 and 1932 at Hawthorne Works, a factory outside of Chicago, Illinois. Company leaders wanted to study how levels of light in the workspaces influenced productivity. The workers' productivity increased in both high and low lighting but decreased when the study concluded. Other studies at Hawthorne showed similar change patterns, where improvements existed until the study ended. The workers seemed to be motivated by the studies, not necessarily the environmental changes that were part of the intervention. In 1958, Henry A. Landsberger, a sociologist who studied industrial and labor relations, analyzed the data from the Hawthorne studies and coined the term Hawthorne effect to mean short-lived increases in productivity. Even though this phenomenon has been studied for over seven decades, there is a need for empirical studies to identify the magnitude, conditions, and mechanisms of the Hawthorne effect. ${ }^{12}$

\section{Compensatory Rivalry}

This threat to internal validity occurs in studies using more than one group. Participants aware of the group design may become competitive. Those in the control group, for example, might improve in the outcome measure even though they were not exposed to the intervention due to rivalry with the other group. This is sometimes called the John Henry effect. John Henry is a folk-tale hero. In the story, he was a strong, African American railroad worker. When he became aware his work was being compared to that of a steam drill, he put forth so much extra effort that the added exertion killed him. ${ }^{1}$ Compensatory rivalry in research studies is not just folklore. Consider a worksite wellness intervention to facilitate weight loss. Participants in the control group may work harder to lose weight due to competition with their coworkers, who are assigned to the intervention group. Getting input from the sample population during planning can help the research team develop strategies to minimize the potential for this threat to internal validity.

\section{Compensatory Demoralization}

When people are assigned to the control group instead of the intervention group, they may feel resentful or disadvantaged. This, in turn, may affect their commitment to the study and the level of differences in outcome between the groups. Dropout rates may also increase in participants with feelings of compensatory demoralization. Imagine a study on cognitive benefits of exercise in older adults. One group gets private sessions with a personal trainer while they work out on an exercise bicycle and one group follows a self-guided series of stretches. The room in which they complete the stretching has a big glass window into the area of the exercise bicycles. The stretching group can see the bicycle group having fun, while they lie on the floor and stretch. The stretching group feels demoralized for not being chosen 
for the intervention group. Even if all participants get a free gym membership after the study ends, those in the stretching group may become less engaged and more likely to drop out of the study.

\section{THE PLACEBO EFFECT}

Placebos are treatments that have no effect (e.g., sugar pill), but that are given to participants to make them think they are being treated. A placebo effect occurs when participants receiving a placebo show improvement in the outcome compared with participants who receive no treatment (i.e., no placebo or intervention). Psychological outcomes, such as perceived effort or pain tolerance, are more vulnerable to placebo effects than physiological outcomes. "Blinding" is sometimes used in placebo studies. In blinded studies, participants do not know whether they are receiving the treatment or the placebo until the study ends. In double-blinded studies, both the researchers and the participants do not know who is receiving the treatment and who is receiving the placebo. The use of a placebo control group can also be a way to differentiate the impact of placebo effect on internal validity. If the risk for placebo effect is high, a researcher would design a study with three groups: an intervention group (receiving treatment), a placebo group (receiving a placebo), and a control group (receiving no treatment or placebo). The use of placebos is more common in medical research than in public health research. However, the concept of "the placebo" can be integrated into public health studies in some cases. Crum and Langer ${ }^{2}$ conducted a study on the health of 84 female hotel cleaners. They told half of the participants that the work they did counted as exercise and they were active enough to achieve the recommended amounts of physical activity as per the U.S. physical activity guidelines. The other half of the participants were provided with no information other than they were participating in a health study. Although their behavior did not change during the 4-week intervention, the informed group perceived themselves as getting more exercise than at baseline. They also showed improvements in weight, body mass index, blood pressure, and waist-to-hip ratio. ${ }^{2}$ We know from Chapter 1, The Importance of Research in Public Health, that one small study does not provide conclusive evidence on the efficacy of placebo effect on exercise, but it is a unique, nonmedical example of a placebo study.

\section{EXPERIMENTAL STUDY DESIGNS}

The features within study designs define the extent to which we can determine whether the change in our outcome of interest (dependent variable) was due to our independent variable. Experimental study designs have features providing the highest level of control for threats to internal validity, while meeting the three criteria for inferring causality. In experimental studies, participants are randomly assigned to either an experimental group or a control group. Each group is assessed, the experimental group receives the intervention or treatment, and both groups are reassessed. The results of the outcome of interest are compared between 
the two groups. The classic experimental study design is the pretest-posttest control group design. This design can be drawn as follows:

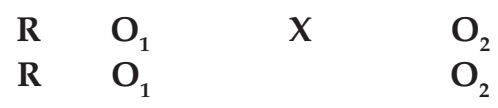

In this depiction, $\mathbf{R}$ stands for random assignment into intervention or control groups, which is a hallmark of the experimental design. $\mathbf{O}_{1}$ stands for pretest or baseline measures. The $\mathbf{X}$ is the intervention or treatment (not given to control group). $\mathbf{O}_{2}$ stands for posttest. These notations are commonly used to outline study designs. The pretest-posttest study design is also shown in Exhibit 6.1.

This design has features to control for threats to internal validity. Look at the study example in Exhibit 6.1. Selection bias is reduced or eliminated through random group assignment. The groups of adolescents should be comparable. Random selection also makes it less likely that one group would be more likely to statistically regress from extreme score to mean scores than the other group. Any changes in stress perception due to maturation would be seen in both groups. Pretest-posttest control group design is common in public health research. See Table 6.1 for some examples. One threat to internal validity that may be a concern when using this study design is testing. A posttest-only control group design eliminates the impact of the testing effect. This design is similar to pretest-posttest control group design, but without the pretest. It can be depicted as:

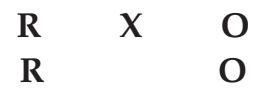

What if in order to answer your research question, you still need to determine differences between pre- and posttest measures, but there is the potential threat of a testing effect? You could use a Solomon four-group study design. This combines the classic pretest-posttest control group design with the posttest-only control group design. All groups are randomly assigned, but two get the intervention; one intervention group with pretest, and one intervention group without pretest. This design also has two control groups: one control group with

\section{EXHIBIT 6.1 BASIC EXPERIMENTAL DESIGN: PRETEST-POSTTEST CONTROL GROUP}

\begin{tabular}{|c|c|c|}
\hline Experimental Group & & Control Group \\
\hline Pretest & ------ compare----- & Pretest \\
\hline (Intervention) & & \\
\hline Posttest & ------ compare----- & Posttest \\
\hline
\end{tabular}




\begin{tabular}{|l|l|}
\hline TABLE 6.1 EXAMPLES OF PRETEST-POSTTEST CONTROL GROUP DESIGNS IN \\
PUBLIC HEALTH RESEARCH \\
\hline Citation & Description \\
\hline Swartz et al. ${ }^{3}$ & $\begin{array}{l}\text { Randomized to intervention group of students and waitlist } \\
\text { controls for depression literacy. Used pretest assessment and a } \\
\text { 4-month follow-up. }\end{array}$ \\
\hline Mutanski et al. ${ }^{4}$ & $\begin{array}{l}\text { HIV-negative men randomly assigned to eHealth intervention or } \\
\text { control condition. Baseline assessment and postdata collected } \\
\text { 3, 6, and } 12 \text { months after intervention. }\end{array}$ \\
\hline Garrison et al. ${ }^{5}$ & $\begin{array}{l}\text { Participants assigned to intervention or usual experience } \\
\text { and measured smoking abstinence } 1 \text { week and } 6 \text { months } \\
\text { postintervention. }\end{array}$ \\
\hline Crosby et al. ${ }^{6}$ & $\begin{array}{l}\text { Participants were randomized to interactive intervention or } \\
\text { attention-equivalent control condition. Assessments occurred at } \\
\text { baseline, } 2, \text { and } 6 \text { months after intervention. }\end{array}$ \\
\hline Chang et al. ${ }^{8}$ & $\begin{array}{l}\text { Women aged } 18 \text { to } 25 \text { were randomly assigned to 14-day } \\
\text { intervention or diet-as-usual conditions. Depression and anxiety } \\
\text { were measured pre- and postintervention. }\end{array}$ \\
\hline $\begin{array}{l}\text { Participants randomized to community health worker } \\
\text { intervention or usual care. Pretest measures and posttest } \\
\text { performed on access and utilization after } 1 \text { year. }\end{array}$ \\
\hline 7
\end{tabular}

pretest, and one control group without pretest. If testing influences change the outcome, you would find differences when comparing the experimental groups with each other as well as comparing the results between the two control groups. The Solomon four-group design can be drawn as:

$\begin{array}{llll}\mathbf{R} & \mathrm{O}_{1} & \mathrm{X} & \mathrm{O}_{2} \\ \mathbf{R} & \mathrm{O}_{1} & & \mathrm{O}_{2} \\ \mathbf{R} & & \mathrm{X} & \mathrm{O}_{2} \\ \mathbf{R} & & & \mathrm{O}_{2}\end{array}$

Suppose you are interested in the effects of an intervention to increase safe-sex practices among college students. The intervention consists of a 2-hour peer-led information session on sexual health. You want to get a baseline assessment of knowledge about sexual health and safe-sex practices, but past literature indicates high risk for testing effects in this type of study. During student orientation, you randomly assign students to four groups and provide 
the intervention to two of the four groups. You conduct a pretest in two groups (one experimental and one control) and, after 30 days, collect posttest data from participants in all four groups. Your analysis of group results shows significant differences in safe-sex practices among the groups with and without the pretest. You conclude that it was not the intervention, but the pretest, that influenced changes in safe-sex practices.

Public health interventions can be complex and multifaceted. For interventions having multiple components, researchers may be interested in studying the effectiveness of each component separately, or different combinations of components. Maybe the whole intervention is not needed to create the desired outcome. Resources can be used more judiciously if there is evidence on the most efficient ways to deliver interventions. Dismantling study designs look at the whole intervention, but also break down the intervention components. This type of design is depicted as:

$\begin{array}{llll}R & \mathrm{O}_{1} & \mathrm{X}_{\mathrm{AB}} & \mathrm{O}_{2} \\ \mathbf{R} & \mathrm{O}_{1} & \mathrm{X}_{\mathrm{A}} & \mathrm{O}_{2} \\ \mathbf{R} & \mathrm{O}_{1} & \mathrm{X}_{\mathrm{B}} & \mathrm{O}_{2} \\ \mathbf{R} & \mathrm{O}_{1} & & \mathrm{O}_{2}\end{array}$

Here, $\mathbf{A B}$ stands for the whole intervention, and both $\mathbf{A}$ and $\mathbf{B}$ separately depict separate intervention strategies or components. As in the other experimental designs, a control group gets no intervention. This type of study design is becoming increasingly important in public health research - technological advances are providing more options for intervention delivery. Let us use as an example a nutrition intervention with two components. The intervention consists of an in-person series of classes coupled with weekly online modules. With dismantling, the researcher can compare the effectiveness of the whole intervention, the in-person component and the online component.

Another experimental study design important for public health research is called the alternative treatment design. This design is similar to dismantling, but only separate components (not the components together) are investigated. Alternative treatment design is drawn as:

$\begin{array}{llll}\mathbf{R} & \mathrm{O}_{1} & \mathrm{X}_{\mathrm{A}} & \mathrm{O}_{2} \\ \mathbf{R} & \mathrm{O}_{1} & \mathrm{X}_{\mathrm{B}} & \mathrm{O}_{2} \\ \mathbf{R} & \mathrm{O}_{1} & & \mathrm{O}_{2}\end{array}$

The intervention or strategies are represented by $\mathbf{X}_{\mathrm{A}}$ and $\mathbf{X}_{\mathrm{B}}$. Let us say you have two feasible intervention options for fall prevention among community-dwelling older adults. $\mathbf{X}_{\mathrm{A}}$ represents an intervention in which health educators make home visits to suggest environmental modifications to reduce risk of falling. $X_{B}$ represents an intervention in which participants get the same information in a group setting at the local library. You collect data on home risk for falls at baseline and again at posttest for all three groups. You study the different interventions or "alternative treatments" separately and compare them to no intervention (control group).

The decision on which experimental design to use should be based on several factors. These questions may help you decide: 
- What is the purpose of my research?

- Which design will provide results to answer my research question?

- What threats to internal validity do I anticipate?

- What lessons learned or recommendations can be found in the literature about my topic of study?

- What study design is feasible and can be implemented with my available resources?

\section{THE ETHICAL CHALLENGE OF CONTROL GROUPS}

Is it ethical to withhold potentially beneficial strategies or services from participants in control groups for the sake of developing evidence? The control group is a hallmark of experimental study designs, but control group participants may be at a disadvantage if the intervention shows effectiveness. This remains an ethical issue in many public health and medical studies. For pharmaceutical studies, the U.S. Food and Drug Administration specifically outlines the types of control groups to be used and when they are appropriate. ${ }^{9}$ There have been cases in which the experimental drug is so effective, the study is halted and control group participants get the experimental drug..$^{10}$

It important to balance the rigor of using control groups in experimental studies with ethical research practices. Researchers may opt to use a wait-list control group (where the intervention is given to the control group after a period of time). This provides data on the treatment effects, but also allows all participants to gain potential benefits of the intervention. Institutional review boards will identify potential ethical issues with study design and require changes before study implementation. Know the research topic well before designing your study. Look to past studies for control group best practice recommendations.

\section{CHAPTER DISCUSSION QUESTIONS}

1. What factors would you consider when designing a study to answer the research question: "How effective is XYZ intervention in reducing preterm birth among low-income pregnant teens?"

2. Discuss how sample size might affect internal validity.

3. What public health research topics might not be appropriate or ethical for random assignment of study participants?

4. What could you do to reduce the threat of compensatory rivalry and compensatory demoralization? 


\section{RESEARCH PROJECT CHECK-IN}

\section{Causality, Validity, and Design}

Think about the potential threats to internal validity for your proposed study. What are ways you could reduce the potential for these factors? How did past studies on similar topics or within similar populations address these threats? How might you design your study to best answer your research question or achieve the study aim? Even if you are not planning to conduct an experimental study design, it is vital to understand the parameters of causality. This knowledge will help you critically analyze findings from other studies throughout your time as a student, and well into your public health career.

\section{PUBLIC HEALTH RESEARCH METHODS IN REAL LIFE}

\section{A Causality for Concern}

If there was one thing Natalicio remembered from his public health research methods class it was "correlation is not causation." This message was repeated over and over again to get students to understand that just because things are related in some way, we cannot say $X$ caused $Y$ unless certain parameters were met. Natalicio was asked to look into the literature and make recommendations for designing a study. He worked for a large hospital system in the division of employee health. Employee data showed a recent spike in the prevalence of injuries among patient transport workers. His boss wanted to implement an evidence-based intervention on safe lifting and back health with the patient transport workers. Before the hospital administration would sign off on the investment in the intervention for all patient transport workers, they wanted proof that it would reduce the injury rate among this population.

Natalicio looked to the published literature on the intervention to get some ideas for the proposed study design. There were several papers published available because it was the subject of a national multisite randomized control trial. The program was implemented in three large hospital systems in three different states. Patient transport workers at each site were randomized into the intervention group (where they took part in a four-session educational program on safe lifting and back health) or a control group (where they received no education). The program resulted in a significant decrease in injuries among patient transport workers in the intervention group compared with those in the control group at all three sites. However, the articles brought up a few threats to internal validity. First, the workers in both groups worked at the same place and often socialized during breaks and before they clocked in. At one of the research 
sites, it was anecdotally reported that some of the workers in the control group felt as if they were disadvantaged because they were not in the group that received the training on injury prevention. Data from another site showed a decrease in the injuries among workers in the control group. The difference between the intervention and control group at that site was still statistically significant but pointed to a potential Hawthorne effect. All sites reported attrition in program attendance.

Natalicio made note of all of the information reported in the articles so he could propose a study design that used the best possible methods to account for potential threats to internal validity. He also wanted to make sure he was using an experimental design that met the criteria for causality. He designed a pretestposttest control group study for the patient transport workers at the hospital. He thought about ways to reduce interactions with the workers in the control group. One way was to separate them by shift. The rates of injuries were similar in those who worked the morning shift compared to those who worked in the afternoon. He decided to randomly select workers on the afternoon shift to receive the intervention, and randomly select workers from the morning shift for the control group. He made sure the groups were similar in characteristics for comparability. Workers on the afternoon shift were told that everyone would eventually be required to complete the safe lifting and back health program, but administration was implementing it in smaller groups. Control group participants or any of the morning-shift workers received any communication about the program. In order to combat attrition, the program was offered during the workday as part of the normal 8-hour shift. Posttest measures would be prevalence of injuries from patient transport at 4 weeks, 12 weeks, and 6 months after the program.

Natalicio took the study-design plan to his boss for approval. Although it was not perfect, his boss felt it was rigorous enough to demonstrate the effectiveness of the intervention among patient transport workers. They could use the results to gain administration support for full-scale implementation.

\section{Critical Thinking Questions}

1. What are the advantages of duplicating an intervention that is well represented in the literature?

2. What other experimental designs would have worked with this population?

3. Contamination between the intervention and control group was reported as a potential threat. What are other ways Natalicio could have designed the two groups to reduce this contamination?

4. What if the demographics of the patient transport workers were significantly different in Natalicio's hospital compared to what was reported in the literature?

5. Was it ethical to withhold the intervention to the control group if evidence showed it could reduce injuries? Why or why not? 


\section{COUNCIL ON EDUCATION FOR PUBLIC HEALTH FOUNDATIONAL KNOWLEDGE AND COMPETENCIES}

Foundational Knowledge

Profession and Science of Public Health

- Explain the critical importance of evidence in advancing public health knowledge.

Foundational Competencies

Planning and Management to Promote Health

- Select methods to evaluate public health programs.

\section{REFERENCES}

1. Nelso SR. Steel Drivin' Man: John Henry, the Untold Story of an American Legend. 1st ed. New York: Oxford University Press; 2006.

2. Crum AJ, Langer EJ. Mind-set matters: exercise and the placebo effect. Psychol Sci. 2007;18(2):165171. doi:10.1111/j.1467-9280.2007.01867.x

3. Swartz K, Musci RJ, Beaudry MB, et al. School-based curriculum to improve depression literacy among US Secondary school students: a randomized effectiveness trial. Am J Public Health. 2017;107:1970-1976. doi:10.2105/AJPH.2017.304088

4. Mutanski B, Parsons JT, Sullivan PS, et al. Biomedical and behavioral outcomes of keep it up! eHealth HIV Prevention Program RCT. Am J Prev Med. 2018;55(2):151-158. doi:10.1016/j.amepre.2018.04.026

5. Garrison KA, Pal P, O'Malley SS, et al. Craving to quit: a randomized controlled trial of smartphone app-based mindfulness training for smoking cessation. Nicotine Tob Res. 2018;22(3):324-331. doi:10.1093/ntr/nty126

6. Crosby R, Charnigo RJ, Salazar LF, et al. Enhancing condom use among black male youths: a randomized controlled trail. Am J Public Health. 2014;104:2219-2225. doi:10.2105/AJPH.2014.302131

7. Connor TS, Brookie KL, Carr AC, et al. Let them eat fruit! The effect of fruit and vegetable consumption on psychological well-being in young adults: a randomized controlled trial. PLoS One. 2017;12(2):e0171206. doi:10.1371/journal.pone.0171206

8. Chang A, Patberg E, Cueto V, et al. Community health workers, access to care, and service utilization among Florida Latinos: a randomized controlled trial. Am J Public Health. 2018;108:1249-1251. doi:10.2105/AJPH.2018.304542

9. United States Food and Drug Administration. Guidance for industry E 10 choice of control group and related issues in clinical trials. 2001. Accessed August 16, 2019. http://www.fda.gov/cder/ guidance/index.htm

10. McMurray JJV, Packer M, Desai AS, et al. Angiotensin-neprilysin inhibition versus enalapril in heart failure. N Engl J Med. 2014;371(11):993-1004. doi:10.1056/NEJMoa1409077 
11. Cram $P$, Fendrick $A M$, Inadomi J, et al. The impact of a celebrity promotional campaign on the use of colon cancer screening: the Katie Couric Effect. JAMA Inter Med. 2003;163(13):1601-1605. doi:10.1001/archinte.163.13.1601

12. McCambridge J, Wilton J, Elbourne DR. Systematic review of the Hawthorne effect: new concepts are needed to study research participation effects. J Clin Epidemiol. 2014;67(3):267-277. doi:10.1016/j. jclinepi.2013.08.015

\section{ADDITIONAL READINGS AND RESOURCES}

Banack HR, Kauffman JS. Does selection bias explain the obesity paradox among individuals with cardiovascular disease? Ann Epidemiol. 2015;25(5):342-349. doi:10.1016/j.annepidem.2015.02.008

Bell M, Kenward MG, Fairclough DL, Horton NJ. Differential dropout and bias in randomised controlled trials: when it matters and when it may not. BMJ. 2013;346:e8668. doi:10.1136/bmj.e8668

Naci H, Soumerai SB. History bias, study design, and the unfulfilled promise of pay-for-performance policies in health care. Prev Chronic Dis. 2016;13:160133. doi:10.5888/pcd13.160133externalicon

Trochim WMK. Web enter for social research methods: hybrid experimental designs. 2006. Accessed August 16, 2019. https:// socialresearchmethods.net/kb/exphybrd.php 